\title{
Síntese e Caracterização de Poliuretanos em Dispersão Aquosa à Base de Polibutadieno Líquido Hidroxilado e Diferentes Diisocianatos
}

\author{
Fernanda M. B. Coutinho, Marcia C. Delpech \\ Grupo de Polímeros, IQ/UERJ \\ Thais L. Alves, Ailton S. Gomes \\ Instituto de Macromoléculas Professora Eloisa Mano, UFRJ
}

\begin{abstract}
Resumo: Foram sintetizados, sob a forma de dispersões aquosas, poliuretanos à base de polibutadieno líquido hidroxilado (HTPB), poli(glicol propilênico) (PPG), ácido dimetilol propiônico (DMPA) e quatro diferentes diisocianatos: diisocianato de tolileno (TDI), diisocianato de isoforona (IPDI), diisocianato de 4,4'-diciclohexil-metileno (HMDI) e diisocianato de hexametileno (HDI). Foram avaliadas as características dos filmes preparados por vazamento das dispersões (cast films), o tamanho de partícula obtido a partir das diferentes formulações e o teor de sólidos totais. As dispersões aquosas, com teor de sólidos variando na faixa de 10 a $58 \%$ e com tamanhos de partícula na faixa de 0,4 a 3,5 $\mu \mathrm{m}$, permaneceram estáveis por períodos superiores a 2 meses.
\end{abstract}

Palavras-chave: Polibutadieno líquido hidroxilado, IPDI, TDI, HDI, $H_{12}$ MDI, poliuretanos, dispersões aquosas.

\section{Polyurethane Aqueous Dispersions Synthesis and Characterization Based on Hydroxy-terminated Polybutadiene and Different Diisocianates}

Abstract: Polyurethane aqueous dispersions, based on hydroxy-terminated polybutadiene (HTPB), poly(propylene glycol) (PPG), dimethylolpropionic acid (DMPA) and four different diisocyanates [tolylene diisocyanate (TDI), isophorone diisocyanate (IPDI), 4,4'-diciclo-hexyl-methylene diisocyanate (HMDI) and hexamethylene diisocyanate (HDI)] were produced. The characteristics of the cast films, the particle size, and the solids content obtained from the different formulations were evaluated. The aqueous dispersions with solids content varying from 10 to $58 \%$ and particle size in the range between 0.4 and $3.5 \mu \mathrm{m}$ were stable for periods of more than 2 months.

Keywords: Hydroxy-terminated polybutadiene, poly(propylene glycol), IPDI, TDI, HDI, HMDI, polyurethanes, aqueous dispersions.

\section{Introdução}

A crescente preocupação com questões e exigências ambientais, atualmente já sob regulamentação legal, vem contribuindo muito para o desenvolvimento de sistemas químicos menos agressivos como é o caso das dispersões aquosas ${ }^{[1]}$. Dentre essas, destacam-se aquelas à base de poliuretano ${ }^{[1-3]}$. Os primeiros tra- balhos sobre o desenvolvimento de dispersões aquosas dessas resinas surgiram no final da década de $60^{[4]}$.

Dispersões aquosas de poliuretano são usadas em diversas áreas industriais como a têxtil, a automotiva, a de calçados e também como adesivos e revestimentos para uma ampla gama de substratos ${ }^{[1]}$. Uma outra importante aplicação dos poliuretanos é no desenvolvimento de membranas, que podem ser uti-

Autor para Correspondência: Fernanda M. B. Coutinho, Instituto de Química, UERJ e IMA, UFRJ, Caixa Postal 68525, CEP: 21945-970, Rio de Janeiro, RJ. E-mail: fern@ima.ufrj.br 
lizadas em recuperação de $\mathrm{CO}_{2}$ do petróleo, remoção de hélio do gás natural e separações de misturas de $\mathrm{O}_{2} / \mathrm{N}_{2}, \mathrm{CO}_{2} / \mathrm{N}_{2}$, entre outras ${ }^{[4-6]}$.

Apesar de a pesquisa de sistemas aquosos à base de poliuretanos ter sido iniciada nos anos 60 , publicações sobre o assunto ainda são bastante restritas, devido às peculiaridades e dificuldades da síntese ${ }^{[7-10]}$.

Como se trata de um sistema relativamente novo, a obtenção de dispersões aquosas a partir de novas combinações de reagentes é uma ampla e interessante área de pesquisa. As principais matérias-primas empregadas na produção dos poliuretanos são os di- ou poliisocianatos e os compostos hidroxilados de baixo ou mesmo alto peso molecular (polióis). Dependendo da aplicação do produto final e do tipo de síntese são também usados agentes de cura, agentes de expansão, catalisadores, aditivos, cargas, etc ${ }^{[4]}$.

Entre os monômeros hidroxilados, podem ser utilizados álcoois de baixo peso molecular, polímeros sintéticos, como poliéteres, poliésteres, policaprolactamas, polímeros naturais como, a celulose e a lignina e até triglicerídeos como o óleo de mamona, dentre outros, de acordo com as características esperadas para o produto final[ ${ }^{[4]}$.

Os diisocianatos podem apresentar diferentes estruturas, tais como aromáticas, alifáticas, cicloalifáticas ou policíclicas. Os sistemas mais relatados na literatura são à base de poliéter ou poliéster dihidroxilados e diisocianatos alifáticos ${ }^{[1-3]}$.

Os poliuretanos, assim como a maioria dos polímeros sintéticos, não são solúveis em água. Por isso, é necessário fazer modificações no processo de obtenção desses materiais para viabilizar a formação de dispersões aquosas estáveis ${ }^{[2]}$.

Para promover a compatibilização das cadeias poliuretânicas com água, deve-se introduzir nas mesmas grupos hidrofílicos, que podem ser provenientes de um dos monômeros ou ainda do extensor de cadeia. A presença de grupos iônicos localizados predominantemente na superfície das partículas dispersas é um dos fatores responsáveis pela estabilização do sistema aquoso ${ }^{[1,9]}$.

A grande flexibilidade na seleção dos reagentes possibilita a obtenção de poliuretanos com características físicas e químicas diversificadas. Há, ainda, a possibilidade de se produzir polímeros lineares ou reticulados, contendo não só ligações uretânicas, que são a base de sua classificação, mas também outros tipos de ligações, provenientes dos extensores de cadeia responsáveis pelo aumento do peso molecular do poliuretano ${ }^{[4,11,12]}$.

O objetivo deste trabalho é produzir dispersões aquosas poliuretânicas, de natureza aniônica, à base de polibutadieno líquido hodroxilado (HTPB). A cadeia hidrofóbica do HTPB dificulta a dispersão do poliuretano em água e, por essa razão, há muito poucos estudos na literatura sobre esses sistemas. Neste trabalho, poliuretanos à base de HTPB foram sintetizados com uma série de diisocianatos, incluindo o diisocianato de tolileno (TDI), de natureza aromática, cuja utilização como monômero em dispersões aquosas não tem registro na literatura. Os demais diisocianatos empregados foram de natureza alifática [diisocianato de isoforona (IPDI), diisocianato de 4,4' -diciclo-hexil-metileno (HMDI) e diisocianato de hexametileno (HDI)].

\section{Experimental}

\section{Materiais}

Os seguintes reagentes empregados na síntese das dispersões aquosas de poliuretano foram usadas como recebidos: dibutil-dilaurato de estanho (DBTDL) (catalisador); poli(glicol propilênico) (PPG) - Voranol 2110 , de peso molecular numérico médio $1300^{[2,3]} \mathrm{e}$ número de hidroxilas $=55,42 \mathrm{mg} \mathrm{KOH} / \mathrm{g}_{\text {poliol }}{ }^{[1]}$, polibutadieno líquido hidroxilado (HTPB), de peso

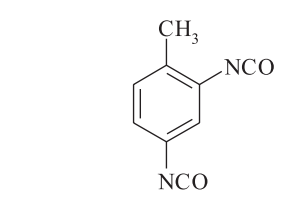

2,4-diisocianato de tolileno (TDI)

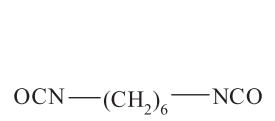

diisocianato de hexametileno (HDI)

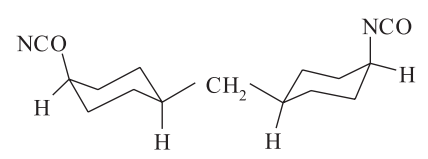

diisocianato de 4,4'-diciclo-hexil-metileno

$\left(\mathrm{H}_{12} \mathrm{MDI}\right)$
Figura 1. Estrutura dos diisocianatos utilizados 
molecular numérico médio $3500^{[12]}$ e número de hidroxilas $=46,06 \mathrm{mg} \mathrm{KOH} / \mathrm{g}$ poliol; ácido dimetilol propiônico (DMPA) (gerador dos sítios aniônicos); trietilamina (TEA) (agente neutralizante para as carboxilas do DMPA); o extensor de cadeia hidrazina (HYD) e os diisocianatos cujas estruturas são apresentadas na Figura 1 [diisocianato de tolileno (TDI), diisocianato de isoforona (IPDI), diisocianato de 4,4'diciclo-hexil-metileno $\left(\mathrm{H}_{12} \mathrm{MDI}\right)$ e diisocianato de hexametileno (HDI)].

\section{Síntese}

Os métodos de síntese dos poliuretanos podem ser classificados em função da ordem de adição dos reagentes e ainda em função do meio físico no qual ocorre a reação. Dependendo do modo de adição dos reagentes, os processos podem ser classificados em processo de uma ou de duas etapas (pré-polímero). Quanto ao meio de preparação, as reações podem ser conduzidas em solução (solventes orgânicos), em dispersão (sistemas aquosos) e em massa (ausência de meio dispersante), sendo esse último o mais utilizado ${ }^{[4,11,12]}$.

Neste trabalho, as sínteses foram conduzidas em duas etapas. A primeira etapa consistiu na preparação do pré-polímero (em massa) e neutralização dos grupos carboxílicos. Na segunda etapa, o prépolímero foi disperso em água e foi, então, conduzida a reação de extensão de cadeia com hidrazina, dando origem assim a dispersões poliuretânicas .

Em balão de fundo redondo, montado em banho de óleo e equipado com agitador mecânico foram adicionados o diisocianato, os polióis (HTPB e/ou PPG), o DMPA e o catalisador. A síntese foi conduzida a $100{ }^{\circ} \mathrm{C}$, por 2 horas, quando os diisocianatos empregados foram TDI, IPDI e HMDI e por 1 hora quando se utilizou HDI. Em seguida foi feita a neutralização com TEA a $45^{\circ} \mathrm{C}$, por 1 hora.

A etapa seguinte foi a dispersão da mistura reacional em água, sob forte agitação, seguida da reação de extensão de cadeia com hidrazina a $35^{\circ} \mathrm{C}$, por 1 hora.

Neste trabalho as composições das misturas reacionais foram feitas de modo a manter o número de equivalentes-grama de compostos hidroxilados fixo e assim distribuído $60 \%$ em equivalentes-grama de DMPA e $40 \%$ de poliol (HTPB e/ou PPG). Foram usadas duas razões ( 2 e 3 ) entre o número de equivalentes-grama do diisocianato e o número de equivalentes-grama total (polióis + DMPA) de compostos di-hidroxilados (razão NCO/OH).
As dispersões poliuretânicas obtidas foram filtradas em tela de nylon ou de aço (mesh 400) para eliminação de partículas grosseiras.

\section{Caracterização}

As dispersões aquosas foram caracterizadas quanto ao teor de sólidos totais, tamanho de partícula e, ainda, quanto às características dos filmes formados. Todos os valores obtidos neste trabalho foram a média de pelo menos duas determinações.

O tamanho de partícula foi determinado por espalhamento de luz laser (LLS) em analisador Malvernsizer Icroplus MAF 5001, em comprimento de onda de $633 \mathrm{~nm}$.

Os sólidos totais, porção não volátil presente nas dispersões, foram determinados adicionando-se $1 \mathrm{~g}$ de dispersão a um vidro de relógio previamente tarado e a massa residual presente nele após secagem em estufa a $100^{\circ} \mathrm{C}$ por 30 minutos foi pesada e relacionada à massa inicial da dispersão. Os valores percentuais foram obtidos multiplicando-se essas razões por 100 .

Foram obtidos filmes das dispersões aquosas por vazamento das mesmas em superfície de teflon, após estas terem sido colocadas em banho de ultra-som por 20 minutos. Os filmes produzidos foram secos à temperatura ambiente por 7 dias.

\section{Resultados e Discussão}

Muitas combinações de reagentes foram empregadas no decorrer deste trabalho, por essa razão a comparação dos resultados obtidos foi feita levandose em conta as dispersões produzidas em uma mesma série, ou seja aquelas obtidas a partir de proporções de reagentes semelhantes, e com variação de apenas um parâmetro reacional.

De um modo geral, na síntese do pré-polímero, o aumento no teor de HTPB resultou na elevação da viscosidade do meio reacional o que dificultou a agitação do sistema.

Outro fator que influenciou acentuadamente o desenvolvimento da reação foi a diferença de reatividade entre os diisocianatos. A reação em que se utilizou o HDI, apresentou um aumento brusco na viscosidade do meio nos primeiros minutos de reação e, por essa razão, o tempo reacional foi reduzido de $2 \mathrm{~h}$ para $1 \mathrm{~h}$.

Como a maior ou menor facilidade de dispersão está ligada à viscosidade do pré-polímero formado, a combinação dos reagentes e ainda o tempo reacional 
tiveram influência sobre a estabilidade das dispersões formadas. Altas viscosidades do pré-polímero levaram a dispersões com baixos teores de sólidos após a filtração da dispersão final. A alta viscosidade dificultou a dispersão e levou à precipitação do poliuretano, em alguns casos, em alta proporção.

Influência do teor de HTPB e da razão NCO/OH sobre o teor de sólidos totais das dispersões aquosas de poliuretanos à base de HMDI

O comportamento das duas famílias de dispersões poliuretânicas à base de HMDI, preparadas nas razões $\mathrm{NCO} / \mathrm{OH}$ igual a 3,0 e 2,0, foi diferente, em relação ao teor de sólidos, quando se variou a proporção de HTPB na formulação (Tabela 1).

As dispersões, preparadas com razão $\mathrm{NCO} /$ $\mathrm{OH}=3,0$, mostraram uma queda acentuada no teor de sólidos com a adição de HTPB (passou de $36 \%$ para $2 \%$ ). No caso das dispersões preparadas na razão $\mathrm{NCO} / \mathrm{OH}=2,0$ pode-se observar que o decréscimo inicial (adição de $10 \%$ de HTPB) do teor de sólidos foi menos acentuado e o aumento posterior da proporção de HTPB (30 \% de HTPB) levou a um aumento do teor de sólidos. No primeiro caso (razão $\mathrm{NCO} / \mathrm{OH}=3,0)$, a viscosidade mais alta do prépolímero dificultou a dispersão do material no meio aquoso, o que provocou uma maior perda de sólidos. Quando se utilizou a razão $\mathrm{NCO} / \mathrm{OH}=2,0$, a viscosidade do pré-polímero foi mais baixa. Isso foi provavelmente devido à maior proporção de PPG que atuou como diluente do HTPB, e assim facilitou a dispersão do pré-polímero em água. O resultado do

Tabela 1. Influência do teor de HTPB e da razão NCO/OH sobre o teor de sólidos totais das dispersões aquosas de poliuretanos à base de HMDI

\begin{tabular}{ccc}
\hline Razão NCO/OH & HTPB/PPG & $\begin{array}{c}\text { Teor de sólidos totais } \\
(\%)\end{array}$ \\
\hline & $0 / 100$ & 25 \\
2,0 & $10 / 90$ & 12 \\
& $30 / 70$ & 31 \\
& $50 / 50$ & $(-)$ \\
\hline \multirow{3}{*}{3,0} & $0 / 100$ & 36 \\
& $10 / 90$ & 2 \\
& $30 / 70$ & $(-)$ \\
& $50 / 50$ & 1 \\
\hline
\end{tabular}

Extensor de cadeia $=$ hidrazina; DMPA $=60 \%$ em relação ao número total de eq.g de compostos hidroxilados, (-) não foi possível obter dispersão estável nessa proporção de reagentes
Tabela 2. Influência do teor de HTPB sobre o teor de sólidos totais das dispersões aquosas de poliuretanos à base de IPDI

\begin{tabular}{cc}
\hline HTPB/PPG & Teor de sólidos totais (\%) \\
\hline $0 / 100$ & 28 \\
$10 / 90$ & 24 \\
$30 / 70$ & 25 \\
$50 / 50$ & 3 \\
\hline
\end{tabular}

Extensor de cadeia $=$ hidrazina; $\mathrm{DMPA}=60 \%$ em relação ao número total de eq.g de compostos hidroxilados; razão $\mathrm{NCO} / \mathrm{OH}=3,0$

teor de sólidos da dispersão preparada com $30 \%$ de HTPB foi inesperadamente alto. Entretanto, a síntese das dispersões com 10 e $30 \%$ de HTPB foram repetidas várias vezes e os resultados foram praticamente os mesmos. Há uma queda do teor de sólidos quando se adiciona $10 \%$ de HTPB e um aumento quando se adiciona $30 \%$ desse poliol. Esse comportamento será investigado em maior detalhe em trabalhos futuros.

Influência do teor de HTPB sobre o teor de sólidos totais das dispersões aquosas de poliuretanos à base de IPDI

As dispersões preparadas com o diisocianato IPDI e com 10 e $30 \%$ de HTPB apresentaram praticamente o mesmo teor de sólidos. Porém, quando se adicionou $50 \%$ de HTPB houve um aumento muito brusco da viscosidade do meio reacional, que dificultou a dispersão e assim causou uma grande perda de sólidos (Tabela 2). O aumento brusco da viscosidade do meio pode ser causado por ocorrência de algum grau de reticulação, pois o HTPB tem uma funcionalidade média próxima de 2 . Contudo, sabe-se que algumas cadeias do HTPB tem funcionalidade maior do que 2, podendo ocorrer, assim, reticulação com o aumento da proporção de HTPB. No caso do IPDI, os grupamentos isocianato estão mais estericamente impedidos, dificultando, desse modo, a reação de reticulação. Quando o teor de HTPB é muito alto, a reticulação passa a ser favorecida pelo fator estatístico, occorendo um aumento brusco da viscosidade com a consequente perda de sólidos.

\section{Influência do tipo de diisocianato no teor de sólidos das dispersões}

A Tabela 3 mostra a variação do teor de sólidos em função do tipo diisocianato empregado, para poliuretanos preparados com razão $\mathrm{NCO} / \mathrm{OH}=3,0$ utilizando apenas PPG como poliol. 
Tabela 3. Influência do tipo de diisocianato sobre o teor de sólidos totais das dispersões aquosas de poliuretanos sem HTPB

\begin{tabular}{cc}
\hline Diisocianato & $\begin{array}{c}\text { Teor de sólidos totais } \\
(\%)\end{array}$ \\
\hline IPDI & 28 \\
HMDI & 36 \\
HDI & 16 \\
\hline
\end{tabular}

Extensor de cadeia $=$ hidrazina; DMPA $=60 \%$ em relação ao número total de eq.g de compostos hidroxilados, razão $\mathrm{NCO} / \mathrm{OH}=3,0 ; \mathrm{HTPB} / \mathrm{PPG}=0 / 100$.

Observou-se que, nessas condições, o diisocianato que levou a maiores teores de sólidos foi o HMDI. Como neste caso não há a influência do HTPB na viscosidade dos pré-polímeros, o fator determinante foi a reatividade do diisocianato. O HDI, que foi o mais reativo dos diisocianatos em questão, uma vez que a etapa inicial da síntese (formação do pré-polímero) ocorreu mais rapidamente, foi o que apresentou os menores valores de teor de sólidos, seguido pelo IPDI, que tem características de reatividade intermediárias entre o HDI e o HMDI.

A Tabela 4 mostra a variação do teor de sólidos em função do tipo de diisocianato empregado, para poliuretanos sintetizados na razão $\mathrm{NCO} / \mathrm{OH}=2,0 \mathrm{e}$ proporção HTPB/PPG =10/90. Nessas condições, o IPDI foi o diisocianato que levou aos maiores valores de teor de sólidos. Novamente, o diisocianato mais reativo, que neste caso foi o TDI, produziu dispersões com menores valores de teor de sólidos.

Não foi possível obter dispersões à base de TDI e HDI nas mesmas condições reacionais, impossibilitando a comparação de resultados entre esses dois diisocianatos.

Influência do teor de HTPB sobre o tamanho médio de partícula das dispersões aquosas de poliuretanos à base de IPDI

A Tabela 5 mostra os resultados das determinações de tamanho médio de partícula das dispersões aquosas.

Tabela 4: Influência do tipo de diisocianato sobre o teor de sólidos totais das dispersões aquosas de poliuretanos preparados em presença de $10 \%$ de $\mathrm{HTPB}$

\begin{tabular}{cc}
\hline Diisocianato & $\begin{array}{c}\text { Teor de sólidos totais } \\
(\%)\end{array}$ \\
\hline IPDI & 19 \\
HMDI & 12 \\
TDI & 10
\end{tabular}

Extensor de cadeia $=$ hidrazina;DMPA $=60 \%$ em relação ao número total de eq.g de compostos hidroxilas, razão $\mathrm{NCO} / \mathrm{OH}=2,0 ; \mathrm{HTPB} /$ $\mathrm{PPG}=10 / 90$
Tabela 5. Influência do teor de HTPB sobre o tamanho médio de partícula das dispersões aquosas de poliuretanos à base de IPDI

\begin{tabular}{cc}
\hline HTPB/PPG & $\begin{array}{c}\text { Tamanho médio de partícula } \\
(\boldsymbol{\mu m})\end{array}$ \\
\hline $0 / 100$ & 0,4 \\
$10 / 90$ & 0,4 \\
$30 / 70$ & 1,2 \\
$50 / 50$ & 3,2 \\
\hline
\end{tabular}

Pode-se observar nessa tabela que, inicialmente, a adição de uma pequena proporção de HTPB (10 \%) não influenciou o tamanho de partícula das dispersões e nem o teor de sólidos. Porém, quando a proporção de HTPB foi aumentada, de $10 \%$ para 30 e $50 \%$, ocorreu um aumento considerável do tamanho de partícula que resultou, no caso extremo de $50 \%$ de HTPB, em um baixíssimo teor de sólidos e um alto valor de tamanho de partícula. Esse resultado deve ser atribuído ao aumento da viscosidade do meio reacional, provocado pelo aumento do teor de HTPB nas formulações, que provocou um decréscimo na velocidade de agitação do sistema reacional, dificultando o mecanismo de quebra das partículas, resultando em partículas finais maiores. As dispersões com muito alto teor (50\%) de HTPB são constituídas por partículas grandes e assim são menos estáveis (grande perda de sólidos).

Influência do teor de HTPB e da razão NCO/OH sobre o tamanho médio de partícula das dispersões aquosas de poliuretanos à base de HMDI

A Tabela 6 mostra os valores de tamanho médio de partícula obtidos nas dispersões à base de HMDI.

Inicialmente (adição de 10\% de HTPB), a natureza mais apolar do HTPB, em relação ao PPG, provavelmente provocou uma maior contração das cadeias poliuretânicas em meio aquoso, em relação às dispersões nas quais o PPG foi o único poliol empregado, provocando, assim, um decréscimo no tamanho médio de partícula. Quando se aumentou o teor de HTPB para além de $10 \%$, ocorreu um aumento acentuado da viscosidade do meio reacional. Isso dificultou o mecanismo de quebra das partículas, através de colisões no meio reacional, e facilitou o mecanismo de coalescência, verificando-se assim um aumento no tamanho médio das partículas. De um modo geral, as dispersões constituídas por partículas menores (mais estáveis) apresentaram os maiores teores de sólidos, com exceção da dispersão preparada com razão $\mathrm{NCO} / \mathrm{OH}$ $=2,0$ e proporção de HTPB de $30 \%$. Essa dispersão 
Tabela 6. Influência do teor de $\mathrm{HTPB}$ e da razão $\mathrm{NCO} / \mathrm{OH}$ sobre o tamanho médio de partícula das dispersões aquosas de poliuretanos à base de HMDI

\begin{tabular}{cccc}
\hline NCO/OH & HTPB/PPG & $\begin{array}{c}\text { Tamanho médio } \\
\text { de partícula } \\
(\boldsymbol{\mu m})\end{array}$ & $\begin{array}{c}\text { Teor de } \\
\text { sólidos totais } \\
(\%)\end{array}$ \\
\hline 2,0 & $10 / 90$ & 3,5 & 25 \\
& $30 / 70$ & 1,0 & 12 \\
3,0 & $0 / 100$ & 7,1 & 31 \\
\hline & $10 / 90$ & 3,4 & 36 \\
& $50 / 50$ & 0,5 & 2 \\
\hline
\end{tabular}

Os valores obtidos são a média de 2 determinações que correspondem ao diâmetro médio das partículas da dispersão medidos por meio de Espalhamento de luz; extensor de cadeia $=$ hidrazina; DMPA $=60 \% \mathrm{em}$ relação ao número total de eq.g de compostos hidroxilados.

apresentou um alto teor de sólidos $(31 \%)$ e um alto tamanho de partícula $(7,1 \mu \mathrm{m})$. Esse resultado inesperado precisa ser melhor investigado.

Influência da combinação de reagentes nas características dos filmes obtidos a partir das dispersões aquosas

A Tabela 7 apresenta as características dos fil- mes produzidos por vazamento das dispersões sobre superfície de teflon.

Os filmes obtidos a partir de dispersões sem HTPB se mostraram mais quebradiços do que os preparados com $10 \%$ de HTPB. Isso mostra que mesmo uma pequena quantidade desse poliol é suficiente para tornar os filmes mais flexíveis. Pode-se ainda observar que o HDI, que é linear e alifático, proporcionou uma maior flexibilidade à cadeia poliuretânica do que os diisocianatos com anel alifático (IPDI e $\mathrm{H}_{12} \mathrm{MDI}$ ). Os filmes nos quais o TDI e/ou HTPB participaram da composição, apresentaram cor amarelada. Isso se deve, provavelmente, à oxidação sofrida pelo anel aromático do primeiro e pela cadeia insaturada do segundo ${ }^{[4]}$.

\section{Conclusão}

Foram obtidas dispersões poliuretânicas aquosas com diferentes composiões e características físicas, com teor de sólidos na faixa de 10 a $36 \%$ e tamanho de partícula na faixa de 0,4 a $3,5 \mu \mathrm{m}$, que foram estáveis por períodos superiores a 2 meses.

Tabela 7. Características dos filmes obtidos a partir do vazamento das dispersões aquosas

\begin{tabular}{|c|c|c|c|}
\hline Diisocianato & $\mathrm{NCO} / \mathrm{OH}$ & HTPB/PPG & Características do filme vazado \\
\hline \multirow{4}{*}{ HMDI } & \multirow[b]{2}{*}{3,0} & $0 / 100$ & Filme homogêneo sem defeitos, duro quebradiço, transparente, sem tack. \\
\hline & & $10 / 90$ & $\begin{array}{l}\text { A dispersão não formou filme, todo o solvente evaporou e formou-se uma } \\
\text { película fina e esbranquiçada na superfície do teflon. }\end{array}$ \\
\hline & \multirow[b]{2}{*}{2,0} & $0 / 100$ & Filme homogêneo sem defeitos, transparente, maleável e sem tack. \\
\hline & & $10 / 90$ & $\begin{array}{l}\text { O filme formado apresentou descontinuidade superficial e pouca aderência, } \\
\text { maleável e sem tack. }\end{array}$ \\
\hline \multirow{4}{*}{ IPDI } & \multirow{2}{*}{3,0} & $0 / 100$ & $\begin{array}{l}\text { Filme homogêneo sem defeitos, muito quebradiço, transparente, pouco } \\
\text { aderente à superficie de vazamento e pouco tack }\end{array}$ \\
\hline & & $10 / 90$ & Filme homogêneo, transparente e quebradiço \\
\hline & \multirow{2}{*}{2,0} & $0 / 100$ & $\begin{array}{l}\text { Não foi possível obter dispersão aquosa estável nessa proporção de } \\
\text { reagentes. }\end{array}$ \\
\hline & & $10 / 90$ & Filme transparente, inteiro, baixa adesividade, com separação de fases \\
\hline HDI & 3,0 & $0 / 100$ & $\begin{array}{l}\text { Filme homogêneo sem defeitos, muito maleável, transparente pouco aderente } \\
\text { e com tack. }\end{array}$ \\
\hline TDI & 2,0 & $10 / 90$ & Filme, com alguns defeitos, maleável e amarelado e sem tack \\
\hline
\end{tabular}

*Os filmes foram vazados em superfície de teflon nivelada e secos à temperatura ambiente durante 7 dias; após esse período foram colocados em estufa à $60{ }^{\circ} \mathrm{C}$ por 2 horas. DMPA $=60 \%$ em relação ao número total de eq.g de compostos hidroxilados. extensor de cadeia $=$ hidrazina 


\section{Agradecimentos}

Ao CNPq e à FAPERJ, pelo apoio financeiro, à COPPE por permitir o uso do equipamento para determinação do tamanho médio de partícula (Malvernsizer), à Petroflex pela doação do HTPB e do IPDI e à Bayer pela doação do HMDI.

\section{Referências Bibliográficas}

1. Coutinho, F. M. B. \& Delpech, M. C. - Polym. Test., 15, p.103 (1996).

2. Coutinho, F. M. B.; Delpech, M. C \& Alves, L. S. - J. Appl. Polym. Sci., p.49-55 (2000)

3. Bamford, C. H. \& Eastmond, G. C. - "Polyurethane Block Copolymers" em Encyclopedia of Polymer Science and Engineering, Mark, H.F.; Bikales, N.M.; Overberger, C.G. \& Menges, G. Jonh Wiley \& Sons, New York, Vol.1, 1985, ps.243-270.

4. Backus, J. K.; Blue, C. D.; Boyd, P. M. \& Squler, E. P. "Polyurethane" em Encyclopedia of Polymer Science and Technology, Raimond B. Seymour, John Wiley, London, V.11, 1987.
5. Huang, S. L.; Chao, M. S. \& Lai, J. Y.- Eur. Polym. J., 34 , p. 469 (1997)

6. Huang, S. L.; Chao, M. S. \& Lai, J. Y.- Eur. Polym. J., 34, p. 449 (1997)

7. Delpech, M. C.; Coutinho, F. M. B.; Moura, P. M. Q. \&. Mello, S. D. S - Polímeros: Ciência e Tecnologia, 3, p. 30 (1996)

8. Kim, C. K. \& Kim, B. K. - J.Appl. Polym. Sci., 43, p. 2295 (1991)

9. Dietrich, D. \& Rieck, J. N. - Adhesives Age, Feb., P. 24 (1978)

10. Nomula, S. \& Cooper, S. L. -'Macromolecules, 33, p. $6402(2000)$

11. Coutinho, F. M. B.; Delpech, M. C. \& Alves, L. S. Polímeros: Ciência e Tecnologia, 10, p. 49 (2000).

12. Coutinho, F. M. B.; Delpech, M. C. \& Alves, L. S. Journal of Applied Polymer Science, 80, p. 566 (2001)

Recebido: 14/02/02

Aprovado: 08/08/02 